\title{
Formas de tratamiento nominales en el discurso escrito en medios virtuales: una aproximación a su ocurrencia según sexo y grupo etario en hablantes de la provincia de Iquique (Chile)
}

\section{Forms of nominal treatment in written digital discourse: an approach on its occurrence according to sex and age group in speakers from the Iquique province (Chile)}

\author{
VIOLETA CAUTÍN-EPIFANI ${ }^{1}$ \\ JADRANKA GLADIC MIRALLES ${ }^{2}$ \\ Pontificia Universidad Católica de Valparaíso \\ violeta.cautin@gmail.com \\ Pontificia Universidad Católica de Chile. \\ Correo electrónico: jagladic@uc.cl
}

Este artículo da cuenta del empleo de las formas de tratamiento nominales (FDTN), en una muestra de discurso escrito en medios virtuales de hablantes de la provincia de Iquique (Chile). Específicamente, se presenta una clasificación de los tipos de FDTN, según sexo y grupo etario en una muestra estratificada de 5.029 INTERACCIONES $^{3}$ publicadas en las Biografías FACEBOOK de 90 sujetos provenientes de Iquique, por un período de 30 días. Los resultados constatan un alto uso de FDTN en este tipo de interacciones y diferencias según sexo y grupo etario de los hablantes.

Palabras clave: Formas de tratamiento nominales, apelación, discurso en medios virtuales, Facebook.

\footnotetext{
${ }^{1}$ Becaria PROGRAMA CONICYT 21100900.

${ }^{2}$ Becaria PROGRAMA CONICYT 22110119.

${ }^{3}$ Entendemos interacciones como "cada uno de los mensajes publicados en una Biografía Facebook, ya sea por el dueño de la correspondiente Biografía o sus amigos Facebook y su correspondiente cadena de comentarios. En este sentido, las interacciones detentan longitudes variables en cuanto a número de palabras ya que algunos mensajes alojaron más comentarios que otros" (Cautín-Epifani, 2015b:69)
} 
This paper gives an account of the use of nominal address forms, in a sample of written digital discourse, of speakers from Iquique (Chile). Specifically, a classification of nominal address forms is presented in a stratified sample of 5.029 INTERACTIONS published in the FACEBOOK Timeline of 90 subjects from Iquique, for a period of 30 days. The results show a high use of nominal address forms in this type of interactions and differences according to sex and age group of the speakers.

Key words: Nominal address forms, address, digital discourse, Facebook.

\section{INTRODUCCIÓN}

Entendemos las fórmulas de tratamiento (FDT) como unidades lingüísticas usadas por los emisores para designar a sus interlocutores (Kerbrart-Orecchioni 2010). Estas formas, además de detentar una función deíctica, se utilizan para establecer el nexo socioafectivo entre los interlocutores (Charadeau y Maingueneau 2005). El español de Chile registra una variedad de FDT que comprende, además de formas nominales, el uso de distintos pronombres (usted, tú y vos) y su correspondiente morfológico (Torrejón 1986; Valencia 2006; Stevenson 2007; Rivadeneira 2009; Bishop \& Michnowicz 2010; CautínEpifani 2015). Teniendo en cuenta que son escasas las investigaciones que indagan sobre el uso de estas formas en la interacción en medios virtuales (excepto Cautín-Epifani 2011; 2015a; 2015b) en el presente trabajo investigamos el uso que se hace de las formas de tratamiento nominales por 90 hombres y mujeres provenientes de la provincia de Iquique, correspondiente a distintos grupos etarios, en una muestra de interacciones publicadas en las Biografía Facebook en un período de 30 días.

Siguiendo a Herring (2001) y Cautín-Epifani y Gladic (en prensa) postulamos el discurso en medios virtuales como toda la interacción entre seres humanos que se efectúa a través de internet, tomando en cuenta sus factores de producción. En este sentido, la interacción en la Biografía Facebook se puede entender como una práctica discursiva semipública que combina elementos de otras prácticas similares, tales como los foros y el chat, pero que se caracteriza por ser un espacio de interacción entre sujetos que, en general, sí mantienen una relación fuera de este espacio virtual ${ }^{4}$.

\section{Marco TEÓRICO}

\subsection{Las formas de tratamiento}

Para el presente estudio, adoptamos la perspectiva teórica sobre las FDT de Kerbrat-Orecchioni. De acuerdo con la autora "Tous les énoncés sont en principe adressés,

\footnotetext{
${ }^{4}$ Para una caracterización más detallada de la interacción en la Biografía Facebook como práctica discursiva ver Cautín-Epifani (2015a) y Cautín-Epifani (2015b).
} 
qu'il comportent ou non un terme d'adresse, c'est-à-dire une forme linguistique designant explicitement l'allocutaire" (Kerbrat-Orecchioni 2010: 9).

Tradicionalmente, las FDT se han agrupado en a) formas pronominales de tratamiento b) formas verbales de tratamiento y c) formas nominales de tratamiento (Braun 1988). Los estudios en FDT para el español de Chile, en general, se han centrado en describir los esquemas pronominales y verbales de trato, los que se ejemplifican en la Ilustración 1. En este sentido, se propone que el sistema de tratamiento pronominal y verbal chileno estaría compuesto por el uso de los pronombres usted, tú y vos, con su correspondiente morfológico. Además, se han identificado formas mixtas que corresponderían al uso del pronombre vos en combinación con una forma verbal tuteante o viceversa (Torrejón 1986) 5 .

\begin{tabular}{|c|c|}
\hline \multicolumn{2}{|c|}{ Tuteo } \\
\hline $\begin{array}{l}\text { Tuteo } \\
\end{array}$ & Ejemplos \\
\hline Tuteo pronominal & ¿Y tú? \\
\hline Tuteoverbal & ¿Cómo estás? \\
\hline Tuteo doble $(\mathrm{pr}+\mathrm{v})$ & ¿Hacia dónde vas tú? \\
\hline \multicolumn{2}{|c|}{ Voseo } \\
\hline Voseo & Ejemplos \\
\hline Voseo pronominal & Vos no. \\
\hline Voseo verbal & ¿Cómo estai? \\
\hline Voseo doble (pr+v) & ¿Hacia dónde vai vos? \\
\hline \multicolumn{2}{|c|}{ Ustedeo } \\
\hline Ustedeo & Ejemplos \\
\hline Ustedeo pronominal & Usted sí. \\
\hline Ustedeo verbal & ¿Cómo está? \\
\hline Ustedeo doble (pr+v) & ¿Hacia dónde va usted? \\
\hline \multicolumn{2}{|c|}{ Formas mixtas } \\
\hline Formas mixtas & Ejemplos \\
\hline Voseo mixto verbal & ¿Tú estai bien? \\
\hline Voseo mixto pronominal & ¿Vos tienes tiempo? \\
\hline
\end{tabular}

Ilustración 1: Formas de tratamiento pronominales y verbales correspondientes al Español de Chile (adaptado de Cautín-Epifani, 2015b)

\footnotetext{
${ }^{5}$ Para una caracterización en profundidad del sistema de tratamiento chileno ver Rivadeneira (2009) y CautínEpifani (2015a)
} 
En la investigación que aquí reportamos, nos centramos, especialmente, en el uso de FDTN entendidas como elementos léxicos que designan al alocutario de la interacción. Las FDTN, al ser utilizadas en el discurso en combinación con los demás tipos de FDT, permiten configurar esquemas de tratamiento que se encuentran determinados no solo en función de las características dialectales de los hablantes, sino también de la situación comunicativa y de los objetivos comunicacionales que persiguen los interactuantes (CautínEpifani 2015a).

A continuación, se detallan algunas de las investigaciones relacionadas con el uso de FDT y sexo, y FDT y grupo etario de los hablantes, así como las principales clasificaciones de FDTN que se han propuesto.

\section{Formas de tratamiento y sexo}

Entre las nociones más generalizadas respecto de la relación entre sexo y uso del lenguaje, se sugiere que las mujeres tenderían a usos lingüísticos que dan preferencia a variantes más prestigiosas, asociadas a la norma culta (García 1999). Cabe señalar que la concepción de lo que se considera como prestigioso varía en función de diferentes factores y no necesariamente todas las comunidades y/o grupos sociales considerarán prestigiosos los usos que se acercan a la norma. Así. afirma Ávila "[las variantes prestigiosas] se relacionan más bien con aquellos elementos que tienen una mayor valoración positiva en una comunidad” (Ávila 2015: 11).

En consecuencia, investigaciones recientes realizadas a partir de datos del discurso oral en medios de comunicación chilenos reportan que tanto en FDT pronominales como verbales, el uso de voseo es más frecuente por parte de hombres que de mujeres. Estas últimas se inclinarían por el uso de variantes más normativas, ya sea ustedeo o tuteo (Rivadeneira 2009; Rivadeneira y Clua 2011; Rivadeneira 2016). Sin embargo, cabe agregar que en situaciones comunicativas menos públicas se ha encontrado que las mujeres igualan e, incluso, superan levemente el uso de voseo (Stevenson 2007; Cautín-Epifani 2015a; Cautín-Epifani \& Rivadeneira, en prensa). Esto se puede relacionar con la interpretación de que el uso de voseo chileno verbal está dotado de prestigio encubierto, ya que su empleo sería muy frecuente entre los jóvenes educados de clase media alta de la capital de Chile (González 2002), y que, además, se encuentra inmerso en un proceso de cambio lingüístico en relación con el sistema de tratamiento pronominal chileno (Rivadeneira 2009), por lo que las mujeres al emplear voseo verbal en contextos de solidaridad estarían participando como propiciadoras de un cambio desde arriba.

Con relación al uso de FDTN y sexo en el español de Chile, las investigaciones son muy escasas. La mayoría de investigaciones se ha concentrado en el uso de palabras altisonantes, tal como la forma huevón (Cortés 2009; Francírková 2012; Rojas 2012), la que se ha reportado como «símbolo de identidad grupal interna» en hablantes hombres (Helincks 2015). Esto reflejaría la tendencia general de las sociedades occidentales a "considerar aceptable o apropiado que los hombres rompan las reglas y que se comporten 
de una manera ruda, agresiva e incluso 'más vulgar'" (Silva-Corvalán 2001: 98). Esto se condice con lo reportado por Cautín-Epifani (2013; 2014a y 2014b) en relación con el uso de vocativos en medios virtuales, quien hace hincapié en la mayor frecuencia de aparición de palabras altisonantes usadas como vocativos por parte de hombres. Por otro lado, no se registran investigaciones que aborden en extenso el uso de FDT y sexo en hablantes chilenos, y menos aún, que se enfoque en las FDTN. En este sentido, la investigación que reportamos provee una primera mirada a esta problemática.

\section{Formas de tratamiento y grupo etario}

Diversos autores coinciden en señalar que la edad es una de las variables que condicionan en mayor medida la variación lingüística (Moreno 2010). Labov (2001) plantea la noción de gradación etaria (age grading), desde la cual se entiende la variación sistemática entre grupos etarios diferentes como un proceso que no involucraría necesariamente un cambio lingüístico, ya que es muy posible que los adolescentes de una época modifiquen con el tiempo el uso que realizan de las diferentes formas lingüísticas para, de ese modo, asemejarse más al habla que sus padres presentarían. Esto debido a que en la adolescencia los hablantes estarían más influenciados por la presión de su grupo social de amistades, quienes buscarían diferenciarse de otros grupos etarios a través de usos lingüísticos particulares. Esta situación cambiaría al llegar a la etapa adulta, pues se insertan en la vida laboral y, al menos en los estratos medios, la presión ahora estaría puesta en ascender socialmente, lo que los lleva a acercarse a las variantes más prestigiosas o tradicionales (Silva-Corvalán 2001).

Ahora bien, Zeller (1997) hace presente que también puede existir el caso en el cual la variación relativa al grupo etario puede haber favorecido un proceso de cambio lingüístico. Esto podría suceder cuando el grupo de menor edad mantiene el uso de una forma lingüística entrada ya a la edad adulta, lo que configuraría un aumento de frecuencia de la ocurrencia de dicho rasgo. Stevenson (2007) plantea para el espańol de Chile que esto podría estar sucediendo con el uso de voseo, el que parece haber aumentado.

Respecto de investigaciones que indagan sobre el uso de las FDT pronominales en el español peninsular, la edad ha sido constantemente señalada como uno de las variables más influyentes. Diversos estudios reportan el mayor uso de la forma pronominal tú en el habla juvenil en diferentes comunidades de España. Estos resultados contrastarían con el habla de los adultos, quienes tienden a preferir la mantención de la forma usted, especialmente, en el trato a personas menos cercanas. En este sentido, el habla adolescente sería menos conservadora (Aguado 1981; Alba y Sánchez 1980; Borrego, Gómez \& Pérez 1978; Criado de Val 1973; Marín 1972).

En relación al español de Chile, Jørgensen (2007; 2011), basándose en datos del corpus COLA, plantea que los adolescentes usarían con mayor frecuencia palabras altisonantes como vocativos, específicamente, huevón/ona, maricónla, cualiadola y otros tales como locola y chicola. 


\section{Clasificaciones de las formas de tratamiento nominales}

Diferentes investigadores han propuesto variadas clasificaciones para las FDTN. Braun (1988) en una investigación que trata de dar cuenta del sistema de tratamiento de las diferentes lenguas en general, sin basarse en un corpus específico, clasifica estas formas en nueve categorías:

Tabla 1. Clasificación de formas de tratamiento nominales según Braun (1988)

\section{Braun (1988)}

\section{Nombres}

2. expresiones de parentesco

3. expresiones generales de tratamiento del tipo señor/señora

4. títulos

5. sustantivos que aluden a una cualidad abstracta del destinatario, tales como $S u$ Excelencia

6. expresiones que denotan la naturaleza de la relación entre los hablantes, tales como colega, amigo

7. expresiones de ocupación, tales como taxista, doctor.

8. expresiones que definen al destinatario por medio de su relación con otra persona, tal como Abu Ali (hijo de Alí en árabe), d Mohammed lur (hija de Mohamed en pashto)

9. expresiones amorosas o de cariño

No podemos dejar de notar en la clasificación propuesta por Braun (1988) la falta de una categoría referida a las expresiones afectivas de valor negativo, tales como palabras altisonantes, presente en la mayoría de las demás clasificaciones revisadas.

Dunkling (1990) en su obra A Dictionary of Epithts and Terms of Address, basándose específicamente en la lengua inglesa, realiza una clasificación en siete categorías: 
Tabla 2. Clasificación de formas de tratamiento nominales según Dunkling (1990)

Dunkling (1990)

1. nombres, subclasificados en:

a) nombres de pila, apellidos y middle names : los que en la lengua inglesa se pueden realizar en las siguientes combinaciones: i) nombre de pila, ii) diminutivo del nombre de pila, iii) nombre de pila y middle name, iv) apellido, v) nombre de pila y apellido, vi) nombre de pila, middle name y apellido, vii) iniciales.

b) apodos: entendidos como cualquier nombre extra, usualmente no oficial, que se utiliza para identificar a una persona en situaciones no formales. i) apodos privados: generalmente utilizados entre personas en una relación romántica y en contextos íntimos, ii) apodos grupales: utilizados por un grupo de personas para identificar al destinatario, iii) apodos públicos: son apodos genéricos utilizados para identificar a una persona como perteneciente a una categoría.

c) nombres transferidos: refieren a la transferencia de un nombre de una persona real o ficticia para identificar al destinatario debido a que este posee caraterísticas compartidas con el personaje nombrado. A diferencia de los apodos, estos nombres no se utilizan con regularidad.

d) nombres substitutos: refieren al uso de un nombre de pila genérico para sustituir el nombre del destinatario, generalmente por desconocimiento de éste.

e) nonce names: refiere al uso de una palabra convertida en nombre de manera temporal para ser utilizada en una determinada persona. Generalmente se utiliza un prefijo para realizar esta conversión, por ejemplo: $M r$ Policeman, $M r$ Lawyer, $M r$ Bigshot, $M r$ Hypocrite, $M r$ Clever, $\mathrm{Mr}$ High-and-Mighty.

f) nombres burlescos (mock names):

g) nombres numéricos,

h) nombres colectivos,

i) nombres de lugar,

\section{2. expresiones de parentesco}

3. expresiones de carińo y amistad

4. términos de tratamiento corteses

5. términos de tratamiento neutrales

6. expresiones insultivas

7. vocativo omitido

Rigatuso (1994; 1997; 2000), en una serie de investigaciones que indagan sobre los nombres de tratamiento en Buenos Aires, ha clasificado estas en siete categorías: 
Tabla 3. Clasificación de formas de tratamiento nominales según Rigatuso (1994; 1997; 2000)

Rigatuso (1994; 1997; 2000)

1. expresiones de parentesco

2. formas generales

3. formas ocupacionales

4. formas de amistad, cordialidad y afecto

5. formas honoríficas

6. nombres de pila o sobrenombres y apellidos

Cautín-Epifani (2015b), en una investigación realizada sobre FDT en una red comunicativa virtual de hombres jóvenes chilenos, realiza la siguiente clasificación (adpatada de Edeso, 2005):

Tabla 4. Clasificación de formas de tratamiento nominales según Cautín-Epifani (2015b)

Cautín-Epifani (2015)

1. palabras altisonantes

2. variaciones del nombre

3. apodos - expresiones adjetivas

4. apodos - expresiones zoonímicas

5. expresiones de parentesco

6. otras expresiones sintagmáticas

Kerbrat-Orecchioni (2010: 21-23), en una investigación basada en un corpus de textos en francés pertenecientes a diferentes géneros discursivos en el ámbito del comercio y debates televisivos, plantea la siguiente clasificación: 
Tabla 5. Clasificación de formas de tratamiento nominales según Kerbrat-Orecchioni (2010)

Kerbrat-Orecchioni (2010)

1. nombres, que incluye apellidos, nombres de pila, diminutivos, sobrenombres y otros de este tipo,

2. formas señor, señora, señorita, los que postula que han perdido su valor de título, sino que son usados como formas de tratamiento generales en relaciones de poca familiaridad,

3. honoríficos, ya sea títulos nobiliarios o conferidos, como capitán, chef, entre otros, siempre y cuando retengan un valor honorífico,

4. nombres de profesión y de función,

5. expresiones relacionales, incluye los términos de parentesco y otras expresiones que explicitan la relación entre los hablantes, como colega, amigo, entre otros,

6. etiquetas, entendidas como expresiones que categorizan al interlocutor y que se usan de manera provisional o improvisada, designándolo a través de, por ejemplo, una sinécdoque a partir de alguna de sus características físicas,

7. expresiones afectivas, incluye formas con valor positivo o negativo, términos relativamente lexicalizados como cheri, mon ange, ma belle, y otras metáforas de base animal o no que pueden ser más o menos estereotipadas o creativas.

En la presente investigación hemos adoptado la clasificación propuesta por Kerbrart-Orrecchioni (2010), por tratarse de la que, a nuestro parecer, da mejor cuenta de las ocurrencias encontradas en nuestro corpus. Además, debido a las limitaciones de la extensión de nuestro estudio, no nos es posible realizar una clasificación más pormenorizada de las ocurrencias de este tipo de formas, tal como la que propone Dunkling (1990), por ejemplo.

\section{Marco metodológico}

La investigación es de carácter descriptivo y se realizó mediante procedimientos de análisis de porcentajes de ocurrencia. Se recolectó un corpus de 5029 interacciones publicadas en las Biografías Facebook de 90 sujetos provenientes de la provincia de Iquique, por un período de 30 días (diciembre de 2011). El corpus se recolectó, con autorización de los sujetos dueños de las Biografías Facebook, entre los meses de abril y mayo del año 2013. $\mathrm{Al}$ momento de producirse las interacciones ni los sujetos dueños de las Biografías Facebook, ni las personas comentaristas tenían conocimiento de que sus interacciones serían objeto de estudio posterior.

La selección de informantes se realizó siguiendo los criterios de muestreo por cuotas con afijación uniforme pre-estratificada en función de las variables sociales de sexo y edad. 
Otros criterios de selección corresponden a la residencia en la provincia de Iquique por al menos siete ańos previos a la producción de las interacciones y al nivel de escolaridad'. A continuación, se presenta la grilla final con las características de los informantes cuyas intervenciones componen el corpus:

Tabla 6. Caracterización de los sujetos informantes

\begin{tabular}{|l|c|c|c|c|c|c|}
\hline \multicolumn{1}{|c|}{ Grupo etario } & \multicolumn{2}{|c|}{$\begin{array}{c}\text { 14 a 17 años } \\
\text { (Grupo 3) }\end{array}$} & \multicolumn{2}{c|}{$\begin{array}{c}\text { 20-26 años } \\
\text { (Grupo 2) }\end{array}$} & \multicolumn{2}{|c|}{$\begin{array}{c}\text { 30-36 años } \\
\text { (Grupo 1) }\end{array}$} \\
\hline Ocupación & \multicolumn{2}{|c|}{$\begin{array}{c}\text { estudiante colegio } \\
\text { particular-subvencionado }\end{array}$} & $\begin{array}{c}\text { estudiante } \\
\text { universitario }\end{array}$ & \multicolumn{2}{c|}{$\begin{array}{c}\text { profesional } \\
\text { universitario }\end{array}$} \\
\hline Procedencia geográfica & \multicolumn{7}{|c|}{ Provincia de Iquique } \\
\hline Sexo & $\mathrm{H}$ & $\mathrm{M}$ & $\mathrm{H}$ & $\mathrm{M}$ & $\mathrm{H}$ & $\mathrm{M}$ \\
\hline Número de sujetos & 15 & 15 & 15 & 15 & 15 & 15 \\
\hline
\end{tabular}

La metodología de análisis tuvo dos instancias. En primer lugar, se identificaron todas las FDTN, las que se etiquetaron manualmente en el corpus. Luego, estas fórmulas se clasificaron siguiendo las categorías propuestas por Kerbrat-Orecchioni (2010). En una segunda instancia, se procedió a realizar análisis respecto del porcentaje de ocurrencia de las fórmulas nominales encontradas en el corpus. Así se determinó el porcentaje de ocurrencia de cada categoría en relación a los diferentes subgrupos detallados en la tabla 6 .

\section{Resultados}

Se contabilizaron 1.088 ocurrencias de FDTN, correspondientes al 37,6 \% del total de FDT identificadas en el corpus. Se clasificaron estas ocurrencias en las siguientes categorías (adaptadas de Kerbrat-Orecchioni 2010):

\footnotetext{
${ }^{6}$ Se cauteló que los informantes del grupo de 14 a 17 ańos (en adelante adolescentes) fuesen escolares pertenecientes a establecimientos particulares subvencionados (semi-púbicos) de la región, los informantes del grupo 20-26 años (en adelante universitarios) fuesen estudiantes universitarios o técnico universitarios, y los informantes de 29-36 años (en adelante profesionales) fuesen titulados de carreras universitarias o técnico universitarias y que al momento de producir sus intervenciones hubiesen estado empleados.
} 
Tabla 7. Formas de tratamiento nominales por categoría

\begin{tabular}{|c|c|c|}
\hline & Nro. Ocurrencias & Porcentaje global \\
\hline Nombres personales & 394 & $36 \%$ \\
\hline Expresiones relacionales & 286 & $26 \%$ \\
\hline Expresiones afectivas & 230 & $21 \%$ \\
\hline Etiquetas & 150 & $14 \%$ \\
\hline Nombres de ocupación o profesiones & 17 & $2 \%$ \\
\hline Señor o Señora (+ expresión) & 11 & $1 \%$ \\
\hline
\end{tabular}

A continuación, se presentan ejemplos de las FDTN encontradas en nuestro corpus, clasificadas en las categorías señaladas en la tabla $7^{7}$ :

\subsection{Nombres personales}

En esta categoría se incluyen los nombres, nombres compuestos, diminutivos, entre otros. Algunas características de estas FDT en este medio son el uso minoritario de mayúsculas normativas y la duplicación de letras.

(1.a) muchas gracias raul:) $(\mathrm{H} 2)^{8}$

(1.b) ud sabe valecilla , gracias que ti boni :* AGUANTE LA U!! (H2)

(1.c) Marcos, te salió MUY igual a la Paula , jakajkajkajakajkajkajak xD (M2)

(1.d) paulaa salinas!!! aparecisteee!!!! :D (H2)

(1.e) eeeeeeeeeeeeesssssssssssssmmmmmmmmmmmmmmmmmmmeeeeeeeeeeerrrrrrrrrrrrrrr rrraaaaaaaaaaaaaaaaaaaall11111111ddddddddddddaaaaaaaaaaaaaaaaaaaaaaaaaaaaaaaaaa!!!!!!!!!!! !!!!!!!!!!!!!

\footnotetext{
${ }^{7}$ A continuación se presentan los ejemplos más representativos de las ocurrencias de FDTNs encontradas en el corpus. Por razones de espacio no se incluye una relación detallada de todas las ocurrencias, sin embargo, con mucho gusto las autoras enviarán una copia de la relación de dichas formas a quienes se lo soliciten.

${ }^{8}$ Los códigos que se ańaden a los fragmentos informan sobre el subcorpus desde donde se extrajo el ejemplo. $\mathrm{H}$ corresponde al subcorpus hombres y $\mathrm{M}$ corresponde al subcorpus mujer. Los números corresponden a los subcorpus diferenciados por los siguientes grupos etarios: $1=30-36$ años, $2=20-26$ años, $3=14-17$ años. En cada ejemplo hemos respetado la escritura original. Con el objeto de resguardar la anonimicidad de los datos de los ablantes, en algunos casos se eliminaron etiquetas u otros nombres propios, lo que queda consignado en cada ejemplo con la explicación entre corchetes.
} 
(H2)

(1.f) pucha yamil ke rabia no pude ir pq tuve reunion de tesis con la profe :( (M2)

(1.g) oki karinita! (M2)

(1.h) jaja noo yo no tiro palos a nadie.. si llega, llega xD... pero Maca ya comí chocolates ayer, estaban muy ricos :D mmmm quiero más jej (M2)

(1.i) y que onda esa ley maribel? (M2)

(1.j) Cristian wn te eshe demenos hasta tu hermano fue y vo no fuiste po aweonao ;) (M3)

\subsection{Expresiones relacionales}

En esta categoría se incluyen términos de parentesco y otras expresiones que explicitan la relación entre los hablantes, como colega, amigo, entre otros.

(2)

(2.a) y eso que andaba en una fiesta po compare, ya te dije ya $\$ 20.000$ como minimo :P (H3)

(2.b) sii hermano medio panorama :\$ (H3.6.99)

(2.c) Gracias primita :) (H1.1.2)

(2.d) TQ amiguita. =) (H1.12.23)

(2.e) cuñiiiiiiiiiiiiiiiiii!! como sabrás ese día se le ocurrio celebrar el cumple de la sofiii y alguien tenia que quedarse a limpiar :( juntemonos pronto pero para devorarnos el SUSHIII (M2.10.47)

(2.f) kajskasjaksjaksjkasjaksjaksj xddd mamaaaaa qe eri pesa xd (M3.14.26)

(2.g) jakjakj... si saque el $1^{\circ}$ :P amiga cualquier dia me veras por alla,yo (M3.8.2)

(2.h) ajajjaja vesinaaaaa! tanto qee me ckonose :) (M3.12.5)

(2.i) Feliz con la gala de Amirita en Ballet! Hija eres un regalo de Dios! (M1.10.23)

(2.j) bkn... compadre.. gracias por el link... puta espero alguna vez verlos de nuevo =) $(\mathrm{H} 1.12 .24)$

\subsection{Expresiones afectivas}

En esta categoría se incluyen formas con valor positivo o negativo, términos relativamente lexicalizados y otras metáforas de base animal o no que pueden ser más o menos estereotipadas o creativas.

(3)

(3.a) gracia amor [hipervínculo apelativo ${ }^{9}$ ] por la gran noticia ahora la familia pequeña se agrando ya no somos 4 a ahora son 5 beshos y muchos abrazos y a esperar numas 9 o 8 meses XD

$(\mathrm{H} 2.4 .5)$

\footnotetext{
${ }^{9}$ Entendemos por hipervínculo apelativo "un enlace que se inserta en la interacción con la finalidad de llamar la atención del dueño de la página de perfil designada una especie de gesto electrónico." [...] "Postulamos esta categoría como una forma de tratamiento debido a la naturaleza apelativa que se le confiere al hipervículo en esta red social por la funcionalidad del sistema de dar aviso de la utilización del mismo." (Cautín-Epifani, 2015a: 73)
} 
(3.b) Voy a jugarmela si o si x ti amor $\bullet(H 3.2 .6)$

(3.c) weon lei el mail cuando llegue y decia de 21:10 a 23:30 :s (H1.2.31)

(3.d) ssisisisi wn te gusta wear $\mathbf{q} \mathbf{l}$ xd (H3.6.121)

(3.e) No wna, fue la marta $\mathrm{xD}(\mathrm{H} 3.9 .23)$

(3.f) chanchito wilfridooo! jajaja mi amor si nunca dijieron que se llamaba babe! (M2.10.20)

(3.g) te amo mi vidaaaaa!!!! mi toooooo tan lindo cmo siempre..... (M3.13.42)

(3.h) te amo precioso mío $\$$ ! muaaak ;*** (M2.2.70)

(3.i) jajaja raton de cola largaa le conseguiste a carlitros ? (H1.5.5)

(3.j) jajaja chacalooooo sacate unas heladitas ;D (H1.5.28)

\subsection{Etiquetas}

En esta categoría se incluyen expresiones que categorizan al interlocutor y que se usan de manera provisional o imporvisada, designándolo a través de, por ejemplo, una sinécdoque a partir de alguna de sus características físicas.

(4)

(4.a) Fue un gran día para mi en lo personal =) ... quisiera tomar el primer vuelo hacia el paraíso y llenarte de besos mi vida, porque eres mi vida mujer. (H1.12.22)

(4.b) viejooo, hijo mio yo trabajo hasta el miercoles 7 no mas po, vo cachai po. asi q desde el jueves yo estoy pitFull (H1.2.42)

(4.c) wenaaaa rubiaaa! feliz navidad tambien para ti y tu pololo :D (H2.12.10)

(4.d) estoy vivo bonita, disculpa por no llamarte pero me senté en mi cama y me quede dormido. (H2.14.73)

(4.e) Me qitaste a mi pololo y ahora deci qe te tengo botao 7 ' ashuashaushasusha hablame cuando te conectes niña *.* te qiero mas $(\mathrm{H} 3.14 .52)$

(4.f) No paisana D:! el padre director asuhsahuas csm! (H3.14.47)

(4.g) Gracias amigas!!! chinita te cambiai de casa me contaron, dnd te vai? (M1.13.104)

(4.h) estúpidaaa mira tu correo...... (denissemail....) perdón! hotmail... (M1.6.17)

(4.i)... yap, ahí estaré para verte de viejiiiito POLscuero! , jajajaja, TE AMO buatOm : ${ }^{* * * * * *}$ (M2.2.50)

(4.j) guashi! al principio solo tenia pa hacer ring y de ahi ni pa eso! te hice mil perdidas!! lo intente hasta kmo la s6! (M2.1.26)

\subsection{Nombres de profesión y/o ocupación}

(5)

(5.a) Lo intentaré, profe. gracias por sus palabras. $(\mathrm{H} 1)$

(5.b) Gracias doctora! :D (M1)

(5.c) muchas gracias profesora nosotras igual la queremos mucho (M2) 


\subsection{Señor / Señora / Señorita ( expresión)}

(6)

(6.a) http://es.wikipedia.org/wiki/Iptables\#Destinos_de_reglas Wikipedia. señor. (H1)

(6.b) Palabras censuradas, Grande Compañera el $1 \%$ de sus empresa Sr piñera y se acaba el

PAYASEO DE LA Robaton el lucro y el negocio (H2)

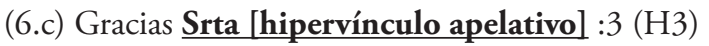

\subsection{Formas de tratamiento nominales por sexo Hombres - Mujeres}

Respecto de las ocurrencias de FDTN por cada categoría en los subcorpus hombres y mujeres, la tabla 8 consigna, de manera decreciente, los porcentajes de ocurrencias de estas. Este análisis permite distinguir, con mayor claridad, las FDTN que más ocurren en cada subcorpus. Tenemos así que en el subcorpus hombres se da preferencia al uso de expresiones afectivas $(36,67 \%)$ y nombres personales $(31,11 \%)$, mientras que entre las mujeres se da preferencia al uso de nombres personales $(39,81 \%)$ y expresiones relacionales $(31,50 \%)$.

Tabla 8. Porcentajes de ocurrencias de formas de tratamiento nominales por subcorpus (hombres y mujeres)

\begin{tabular}{|c|c|c|c|c|c|c|}
\hline & $\begin{array}{c}\text { Nombres } \\
\text { personales }\end{array}$ & $\begin{array}{c}\text { Expresiones } \\
\text { relacionales }\end{array}$ & $\begin{array}{c}\text { Expresiones } \\
\text { afectivas }\end{array}$ & Etiquetas & $\begin{array}{c}\text { Señor o } \\
\text { Señora }\end{array}$ & $\begin{array}{c}\text { Nombres de } \\
\text { ocupación o } \\
\text { profesiones }\end{array}$ \\
\hline Hombres & $31.11 \%$ & $18.89 \%$ & $36.67 \%$ & $10.44 \%$ & $1.33 \%$ & $1.56 \%$ \\
\hline Mujer & $39.81 \%$ & $31.50 \%$ & $10.19 \%$ & $16.14 \%$ & $0.78 \%$ & $1.57 \%$ \\
\hline
\end{tabular}

En general, el análisis por sexo muestra una tendencia de ocurrencia similar por cada categoría en ambos subcorpus. La mayor diferencia se presenta en la ocurrencia de expresiones afectivas, la que corresponde a un 36,67 \% en el subcorpus de hombres y solo a un $10,19 \%$ en el subcorpus de mujeres. Creemos que esto se debe a la inclusión de palabras altisonantes en esta categoría, las que aparecen en función apelativa en mayor cantidad en el subcorpus de hombres $(24,8 \%$ del total de FDTN identificadas en dicho subcorpus), mientras que en el subcorpus de mujeres estas formas tienen una ocurrencia muy minoritaria (4,2 \% del total de FDTN identificadas en dicho subcorpus). Las mujeres, en tanto, favorecerían el uso de nombres personales y expresiones relacionales, en especial términos de parentesco y la forma amiga. 


\subsection{Formas de tratamiento nominales por rango etario}

La tabla 9 presenta los porcentajes de ocurrencia de FDTN por categoría en cada subcorpus separado por rango etario. En general, la tendencia se mantiene con un claro predominio de las ocurrencias de nombres personales, especialmente, en el subcorpus 29-36 ańos con un 45,56 \%. Las ocurrencias de expresiones relacionales presentan un porcentaje muy similar en los subcorpus 29-36 ańos y 20-26 ańos con un 32,03 \% y $31,76 \%$, respectivamente. El subcorpus 14-17 ańos presenta un porcentaje menor en esta categoría con un 16,83\%, lo que contrasta con el porcentaje de ocurrencias en la categoría expresiones afectivas con un 32,92\% en el grupo antes mencionado. Como lo seńalamos anteriormente, en esta categoría se incluyen las palabras altisonantes, lo que creemos tiene relación con este aumento. En la categoría etiquetas se aprecia una relación ascendente de ocurrencias a medida que el corpus representa las interacciones de sujetos de menor edad. Las restantes categorías presentan porcentajes similares, bastante por debajo de las categorías anteriormente comentadas.

En general, no se aprecian mayores variaciones por subcorpus etario, con excepción de lo ya seńalado en cuanto a las ocurrencias de expresiones relacionales y expresiones afectivas en el subcorpus 14-17 años. Estos datos parecen indicar que existiría una transferencia en el tratamiento desde las categorías de nombres personales y expresiones relacionales a expresiones afectivas. En este sentido, se podría argumentar que, mientras los sujetos de 2936 y 20-26 ańos prefieren utilizar nombres personales y expresiones relacionales como formas de tratamiento, los sujetos de 14-17 años darían preferencia a la utilización de formas de tratamiento que detentan un valor afectivo, especialmente palabras altisonantes.

Tabla 9. Porcentajes de ocurrencias de formas de tratamiento nominales por subcorpus (rango etario)

\begin{tabular}{|c|c|c|c|c|c|c|}
\hline & $\begin{array}{c}\text { Nombres } \\
\text { personales }\end{array}$ & $\begin{array}{c}\text { Expresiones } \\
\text { relacionales }\end{array}$ & $\begin{array}{c}\text { Expresiones } \\
\text { afectivas }\end{array}$ & Etiquetas & $\begin{array}{c}\text { Señor o } \\
\text { Señora }\end{array}$ & $\begin{array}{c}\text { Nombres de } \\
\text { ocupación o } \\
\text { profesiones }\end{array}$ \\
\hline Hombres & $31.11 \%$ & $18.89 \%$ & $36.67 \%$ & $10.44 \%$ & $1.33 \%$ & $1.56 \%$ \\
\hline Mujeres & $39.81 \%$ & $31.50 \%$ & $10.19 \%$ & $16.14 \%$ & $0.78 \%$ & $1.57 \%$ \\
\hline
\end{tabular}

\section{Conclusiones}

En esta investigación indagamos acerca del uso de fórmulas de tratamiento nominales (FDTN) en el discurso en medios virtuales, específicamente, en la red social virtual Facebook, en relación con el sexo y grupo etario de hablantes provenientes de la 
provincia de Iquique (Chile). En este sentido, continuamos nuestra línea investigativa acerca de los recursos lingüísticos empleados en medios virtuales por hablantes chilenos y presentamos una primera clasificación de las FDTN empleadas por hablantes del español de Chile en interacciones escritas en medios virtuales.

En relación con el sexo de los hablantes, nuestros datos apuntan a una tendencia similar en el uso de estas formas. La mayor diferencia se encuentra en los tipos de FDTN encontradas. En el subcorpus mujeres los nombres personales es la categoría más frecuente, seguida de las expresiones relacionales. Por otra parte, en el subcorpus hombres las expresiones afectivas serían las FDTN más frecuentes, seguidas de los nombres personales. En las demás categorías se observan frecuencias bastante similares de ocurrencia en ambos subcorpus. Postulamos que la diferencia en la mayor frecuencia de aparición de expresiones afectivas en el subcorpus de hombres se debe al abundante uso que estos realizan de palabras altisonantes como formas de tratamiento, lo que es concordante con investigaciones anteriores en las que se indican estas formas como marcadores de identidad masculina, especialmente, en hablantes iquiqueños (Helincks 2015). En contraposición, las mujeres darían preferencia al uso de nombres personales, lo que es concordante con la noción de que estas prefieren el uso de formas más prestigiosas y tradicionales en sus interacciones, asociadas a la norma (SilvaCorvalán 2001). Esto daría cuenta de que los hombres se permiten un uso del lenguaje más agresivo o vulgar con mayor libertad, al menos en esta práctica discursiva que corresponde a discurso semi-público. Estas afirmaciones deberán ser corroboradas en estudios posteriores que se enfoquen en describir en mayor detalle y de manera cualitativa los usos aquí descritos.

En relación con las ocurrencias de estas formas según grupos etarios, el sub corpus 29-36 años presenta una mayor frecuencia de ocurrencias de nombres personales, mientras que en el grupo de 20-26 años la mayor parte de las ocurrencias se reparten entre nombres personales y expresiones relacionales. Finalmente, en el subcorpus 14-17 años la mayor parte de FDTN encontradas corresponde a expresiones afectivas. Nuevamente atrubuimos este último dato al abundante uso de palabras altisonantes que se registra en el subcorpus $14 \mathrm{a}$ 17 años, lo que concuerda con lo reportado en otras investigaciones respecto del lenguaje adolescente (Jørgensen 2007; 2011). Postulamos que el mayor uso de nombres personales que se encuentra en el subcorpus 29 a 36 años podría deberse a la tendencia a utilizar formas más normativas que se ha observado en los sujetos que se encuentran en los primeros ańos de vida laboral (Silva-Corvalán 2011).

\section{Obras citadas}

Aguado, David. 1981. "Análisis sociolingüístico de tu/usted en los estudiantes universitarios de Bilbao". Letras de Deusto 21 (1): 165-184.

Alba, Vidal y Sánchez, Jesús. (1980). “Tratamiento y juventud en la lengua hablada. Aspectos sociolingüísticos”. Boletín de la Real Academia Española 60: 95-130.

Ávila, Viviana. 2015. Caracterización de los estereotipos sexuales en los adolescentes chilenos: 
un análisis de su comportamiento lingüistico. Tesis de maestría, Universidad de Chile, Santiago, Chile.

Bishop, Kelley y Michnowicz, Jim. 2010. "Forms of address in Chilean Spanish". Hispania 93 (3): 413-429.

Borrego, Julio, Gómez, José y Pérez, José. 1978. "Sobre el tú y el usted”. Studia Philologica Salmanticensia 3: 53-70.

Braun, Friederike. 1988. Terms of address: Problems of patterns and usage in various languages and cultures. Berlin: Morton de Gruyter.

Cautín-Epifani, Violeta. 2013. Una exploración de la estructura del vocativo en interacciones mediadas por computadora. Ponencia presentada ante el XX Congreso Internacional de la Sociedad Chilena de Lingüistica, Universidad Católica de la Santísima Concepción, Concepción, Chile.

Cautín-Epifani, Violeta. 2014a. El uso apelativo del hipervínculo: Una exploración en un corpus de hablantes mujeres en la Biografía Facebook. Ponencia presentada ante el VII Coloquio de la Asociación Latinoamericana Estudios del Discurso, Capítulo Argentina, Universidad de Buenos Aires, Buenos Aires, Argentina.

Cautín-Epifani, Violeta. 2014b. Formas de tratamiento nominales en el discurso escrito de hombres chilenos en una red comunicativa virtual. Ponencia presentada ante el XVII Congreso Internacional de Humanidades, Universidad Metropolitana de Ciencias de la Educación, Santiago, Chile.

Cautín-Epifani, Violeta. 2015a. Formas de tratamiento en interacciones verbales escritas en la Biografia Facebook de hablantes de la provincia de Iquique. Tesis doctoral, Pontificia Universidad Católica de Valparaíso, Valparaíso, Chile.

Cautín-Epifani, Violeta. 2015b. "Poder virtual y formas de tratamiento: una exploración en una red comunicativa virtual”. Revista Forma y Función 28 (1): 55-78.

Cautín-Epifani, Violeta \& Lucía Cantamutto. 2016. El Hipervínculo Apelativo como recurso interactivo en el discurso digital. Ponencia presentada ante el IX Encuentro Nacional de Estudios del Discurso, ALED Chile, Universidad Católica de Temuco, Temuco, Chile.

Cautín-Epifani, Violeta \& Jadranka Gladic. En prensa. "Fórmulas nominales de tratamiento en un corpus de discurso escrito en medios virtuales, de hablantes de la provincia de Iquique (Chile)".

Cautín-Epifani, Violeta \& Marcela Rivadeneira. En prensa. "Variación sociolingüística del voseo verbal chileno en interacciones escritas en la Biografía Facebook". Onomázein.

Charadeau, Patrick \& Dominique Maingueneau. 2005. Diccionario de Análisis del Discurso. Buenos Aires: Amorrortu.

Cortés, Saide. 2009. "Expresiones zoonímicas en el habla popular chilena”. Boletín de Filología 44 (2): 243-261.

Criado de Val, Manuel. 1973. "Los pronombres de cortesía: su tratamiento en español y en otros idiomas". Yelmo 10: 5-10.

Dunkling, Leslie. 1990. A dictionary of epithets and terms of address. Londres: Routledge. 
Edeso, Verónica. 2005. "Usos discursivos del vocativo en español”. Español actual: revista de español vivo 84: 123-142.

Francírcová, Eliska. 2012. Análisis comparativo de los marcadores del discurso interactivos en el habla juvenil de Madrid y de Santiago de Chile. Tesis de maestría. Universita Palackého v Olomouci, Moravia, República Checa.

García, Pilar. 1999. Cómo hablan las mujeres. Madrid: Arco Libros.

González, Carlos. 2002. "La variación 'eríh'/'soi' en el voseo verbal de Santiago de Chile. Un estudio exploratorio". Onomázein 7: 213-230.

Helincks, Kris. 2015. "La forma de tratamiento nominal huevón en Iquique (Chile): análisis empírico de conversaciones cotidianas informales". Onomázein 32: 132-151.

Herring, Susan. 2001. Computer-mediated discourse. En: Schiffrin, Deborah; Deborah Tannen \& Heidi Hamilton. Eds. The Handbook of Discourse Analysis. Oxford: Blackwell Publishers. 612-634.

Jørgensen, Annette. 2007. "Corpus del lenguaje adolescente (COLA)". En Luis Cortés et al. Coords. Discurso y oralidad. Homenaje al profesor José Jesús de Bustos Tovar. España: Arco Libros. 221-229.

Jørgensen, Annette. 2011. "Formas de tratamiento: los vocativos en el lenguaje juvenil de Madrid, Buenos Aires y Santiago de Chile". En: Rebollo, Leticia \& Celia Dos Santos Orgs. As formas de tratamento em português e em espanhol. Variação mudança e funçôes conversacionais. Niterói: Editora da UFF. 127-150.

Kerbrat-Orecchioni, Catherine. 2010. S'adresser à autrui: les formes nominales d'adresse en français. Paris: Université de Savoie.

Labov, William. 2001. Principles of linguistic change. Social factors. Oxford: Blackwell.

Marín, Diego. 1972. "El uso de tú y usted en el español actual". Hispania 55: 904-908.

Moreno, Francisco. 2010. Las variedades de la lengua española y su enseñanza. Madrid: Arco Libros.

Rigatuso, Elizabeth. 1994. Fórmulas de tratamiento y familia en el español bonaerense actual. Bahía Blanca: Universidad Nacional del Sur.

Rigatuso, Elizabeth. 1997. Algunos problemas teóricos en el estudio de las fórmulas de tratamiento. Ponencia presentada en IV Congreso Nacional de Lingüística, Mendoza, Argentina.

Rigatuso, Elizabeth. 2000. "Señora (...) ¿No tenés más chico? Un aspecto de la pragmática de las formas de tratamiento en el español bonaerense”. Revista Argentina de Lingüistica 16: 293-344.

Rivadeneira, Marcela. 2009. El voseo en medios de comunicación de Chile. Descripción y análisis de la variación dialectal y funcional. Tesis doctoral, Universitat Pompeu Fabra, Barcelona, España.

Rivadeneira, Marcela \& Esteve Clua. 2011. "El voseo chileno: una visión desde la variación dialectal y funcional en medios de comunicación". Hispania 94 (4): 680-703.

Rojas, Darío. 2012. "Huevón como marcador del discurso en el español de Chile: huellas de un proceso de gramaticalización". Revista de Humanidades 25: 145-164. 
Silva-Corvalán, Carmen. 2001. Sociolingüistica y pragmática del español. Washington: Georgetown University Press.

Stevenson, Jeffrey. 2007. The Sociolinguistic Variables of Chilean Voseo. Tesis doctoral. University of Washington, Washington, Estados Unidos.

Torrejón, Antonio. 1986. "Acerca del voseo culto en Chile". Hispania 69 (2): 677-683.

Valencia, Alba. 2006. Formas pronominales de tratamiento en Santiago de Chile. En: Sedano, Mercedes; Adriana Bolívar \& Mirtha Shiro. Comps. Haciendo lingüística: homenaje a Paola Bentivoglio. Caracas: Universidad Central de Venezuela. 569-581.

Zeller, Christine. 1997. "The investigation of a sound change in progress". Journal of English Linguistics 25 (2): 142-155. 
